\title{
Peyronie's disease: is it genetic or not?
}

\author{
J. Scott Gabrielsen \\ Department of Urology, University of Rochester Medical Center, Rochester, NY, USA \\ Correspondence to: J. Scott Gabrielsen, MD, PhD. Department of Urology, University of Rochester Medical Center, 601 Elmwood Ave, Box 656, \\ Rochester, NY 14642, USA. Email: scott_gabrielsen@urmc.rochester.edu.
}

\begin{abstract}
Peyronie's disease (PD) is a devastating disease that affects up to $13 \%$ of adult men. While trauma to the erect penis is common prior to onset, it is clear that trauma alone is not sufficient to cause PD. Over the past 40 years, multiple lines of evidence have pointed to a genetic factor that predisposes some men to develop PD. Despite dramatic technological advances in the field of genetics, we have made little progress in identifying the genetic contributors to the disease. This review will critically evaluate the literature concerning the genetics of PD published to date. Particular focus will be placed on study design as much of the data are contradictory yet may still give some insight into the etiology of PD. The challenges in identifying the genetic underpinnings of PD will be discussed along with the potential mechanisms that can overcome these challenges.
\end{abstract}

Keywords: Peyronie's disease (PD); genetics; epidemiology

Submitted Sep 16, 2019. Accepted for publication Oct 29, 2019.

doi: $10.21037 /$ tau. 2019.10 .21

View this article at: http://dx.doi.org/10.21037/tau.2019.10.21

\section{Introduction}

Peyronie's disease (PD) is an acquired fibrosis of the tunica albuginea of the penis, affecting up to $13 \%$ of the adult male population (1). The fibrosis is thought to be due to trauma to the erect penis during intercourse, resulting in a palpable, inelastic plaque that causes curvature of the penis when erect. PD can be devastating as there is often a period of active disease associated with severe pain (2); however, even after the pain has resolved, erectile dysfunction is often present. The disease can be both physically and psychologically debilitating (3). While a variety of treatments are available, these are often of limited efficacy and none are able to completely prevent the disease from occurring or progressing.

Over the past 40 years, studies have linked human leukocyte antigen (HLA) group antigens, single-nucleotide polymorphisms, karyotypic abnormalities, and gene expression changes to $\mathrm{PD}$, yet none have definitively identified the underlying etiology. Indeed, despite significant technological advances in the field of genetics, little progress has been made in our understanding of the genetic underpinnings of the disease. In this review, the data supporting - and disputing - a genetic basis for PD will be evaluated. While genetics alone does not cause PD, it remains likely that some men have a genetic predisposition to developing the disease. Possible reasons as to why the genetic link to PD has been so elusive and how these challenges can be overcome moving forward will be discussed.

\section{Factors supporting a genetic basis for PD}

The distribution of PD among different ethnicities is one of the factors suggesting an underlying genetic basis for the disease. PD is more likely to occur in Caucasian men than in other ethnicities (4). Like other diseases predominantly found in specific ethnicities (e.g., cystic fibrosis, hemochromatosis, sickle cell anemia, beta thalassemia, etc.), the relatively high prevalence suggests the presence of a founder mutation.

Other fibroproliferative diseases, especially Dupuytren's disease (DD) are higher in men with PD than the general population. Like PD, DD manifests as fibrosis and contracture of the palmer fascia. About $20 \%$ of men with DD are found to have PD, and DD is frequently found in families with a high prevalence of PD (5-7). This co-occurrence, the overlap of symptoms, and familial transmission suggest a common genetic predisposition.

Many men with PD can recall a traumatic event that preceded the onset of penile pain and subsequent fibrosis 
and curvature $(2,8)$. It should be noted, however, that most cases of penile trauma-especially severe trauma such as penile fracture-do not result in PD. For example, in a study of Iranian men, no cases of PD occurred among 193 patients who underwent surgical repair for penile fracture (9). Thus, some individuals appear to be primed to develop the disease in response to relatively minor trauma, while others can experience major trauma without developing diseaseas would be expected with a genetic predisposition.

\section{HLA, autoimmunity and PD}

The first genetic link to PD was proposed by Willscher et al. in 1979, who hypothesized that HLA-B group antigens would be associated with $\mathrm{PD}$ given their association with other fibrotic diseases (10). They found HLA-B7 cross-reactive antigens in 7 out of 8 men (88\%) with idiopathic PD. Nyberg et al. subsequently published three pedigrees of families with three generations of father-son transmission of $\mathrm{PD}$, reporting that $89 \%$ of the affected individuals cross reacted with the HLA-B7 antigen (6). Ziegelbaum et al. reported on identical twins with $\mathrm{PD}$, whose father and one of their sons had evidence of PD, all of whom were found to carry the HLA-B40 antigen (of note, the B40 antigen cross reacts with the B7 antigen) (7). These data suggest an autosomal dominant, partially penetrant inheritance pattern to PD.

In contrast to these studies, however, Leffell et al. found no association with any HLA antigen among 28 men with idiopathic PD (11). Rompel and colleagues found no association between the $\mathrm{B} 7$ group antigens in 52 individuals with idiopathic PD, but HLA-A1 and HLA-DQ2 antigen frequencies deviated significantly from the local general population (12). HLA-DQ5 antigens were found in $61 \%$ of 31 men with $\mathrm{PD}$, which was 4.6-fold higher than the rate of the 94 controls (13).

These studies were limited in large part by technology and sample size. Advances in the field subsequently allowed Hauck et al. to investigate HLA class I and II antigens in 154 consecutive PD patients compared to a large number of bone marrow donors and a large national cohort of 14,835 men (14). In contrast to all the prior studies, they found no significant differences in HLA-A, B or C, or HLA-DR group antigens. They further concluded that HLA-DQ2 and HLA-DQ5 were unlikely to be different given the strong linkage disequilibrium in their population (14). It should also be noted the clinical relevance of HLA-DQ2 and HLADQ5 antigen differences reported by Rompel et al. (12) and Nachtsheim et al. (13), respectively, is also unknown as HLA-
DQ antigens are undetectable on cells isolated from both PD plaques and control tunica albuginea (15).

While the early and latter studies seem discrepant, the findings are not necessarily mutually exclusive as the populations investigated were quite different. For example, the Nyberg and Ziegelbaum studies were limited to families with evidence of father-to-son transmission $(6,7)$. The Leffell, Rompel, Nachstsheim, and Hauck studies focused on the general PD population, the majority of whom were not related (11-14). The relevance of this difference is demonstrated by the proportion of men with coincident DD, as the Nyberg study found $78 \%$ of the men with PD also had DD compared to only $11 \%$ in the Leffell study $(6,11)$. It is interesting to note, though, that of the three men with both PD and DD in the Leffell study, two were found to cross-react with HLA-B group antigens, consistent with the Nyberg study.

Several other possibilities may explain the disparate findings. First, sample sizes were relatively small and, thus, the studies were not likely to be sufficiently powered to detect differences. The HLA group antigens served as early markers for different alleles before single-nucleotide polymorphisms and more advanced sequencing technologies were available. Thus, the HLA haplotype itself may not be responsible for $\mathrm{PD}$, but rather is closely linked to the causative allele. In families there would likely be greater linkage disequilibrium between a specific antigen and the nearby DNA than in the general population, potentially explaining why familial studies found similar antigens and the other studies did not. Additionally, selecting controls for the studies is challenging, as unscreened controls from the general population might have had PD. In cases of known non-PD controls, however, they could still have had the genetic predisposition to PD but just did not had exposure to the trauma or other inciting event to cause the disease. Depending on the prevalence of the gene in the population, this could greatly increase the number of individuals needed to achieve statistical power. Finally, it is possible that multiple genes are involved, and therefore, one particular gene may be enriched in a family and detectable within a small number of subjects, but not detectable in a more heterogenous group of people.

Nevertheless, the association with between HLA antigens and autoimmune disease led Ferretti et al. to hypothesize that the immune response could induce PD (16). To test this, they performed allografts of the tunica albuginea in Sprague Dawley rats (an outbred strain, so littermates are genetically different from each other). Compared to rats who received their own tunica (i.e., autografts), those who received tissue 
from a different rat developed scarring, chronic inflammation, localized osteogenesis, and curvature that persisted throughout the 12 weeks of the study-a clinical picture very similar to PD (16). Consistent with a potential immune etiology, mycophenolate mofetil (a potent immunosuppressant used to prevent transplant rejection) was found to attenuate plaque development in a TGF- $\beta 1$-induced model of PD in rats (17).

The relationship between autoimmune disease and PD in humans is less clear, particularly given that PD occurs in the absence of allografts. Nonetheless, Ventimiglia et al. evaluated a cohort of 1,149 consecutive men presenting with sexual dysfunction and found autoimmune diseases in $9.5 \%$ of those presenting with $\mathrm{PD}$ compared to $2 \%$ of those presenting with other primary complaints (18). Ralph et al. reported that autoimmune antibodies are elevated in men with PD compared to age-matched controls (19). Antinuclear antibodies were found in $24 \%$ of affected men compared to $4 \%$ of controls; however, no specific anti-penis antibodies were detected. Anti-elastin antibody titers are elevated in men with PD compared to controls (20).

Contrary to these studies, a recent study published by Pastuszak and colleagues used a large claims database to assess comorbid diseases among men with PD (21). They found that autoimmune diseases were not significantly more prevalent among the nearly 9,000 men with PD compared to men with erectile dysfunction (ED) alone or controls.

As with the HLA studies above, differences in the study populations may be responsible for the disparate findings. In the Ventimiglia study, for example, men were selected from those presenting to an academic andrology clinic complaining of sexual dysfunction, whereas the Pastuszak study used men from a much broader background (i.e., those who saw any type of doctor) $(18,21)$. Thus, ascertainment bias could have resulted in a disproportionate number of men with PD being found to have coincident autoimmune disease (Ventimiglia study), whereas there may not be such an association in the general population (Pastuszak study). Alternatively, the Pastuszak study is dependent upon billing codes, which may not accurately capture actual disease (e.g., congenital penile curvature may be coded as PD, whereas PD may be coded as penile pain, particularly by non-urologists). Neither of these studies looked at HLA antigens or genetic markers. Therefore, even if there is an association between autoimmune disease and PD, there still may not be a genetic link.

\section{Single-nucleotide polymorphisms and PD}

Genome-wide association studies (GWAS) are chip-based arrays that identify single-nucleotide polymorphisms across the whole genome. While these polymorphisms may be directly pathologic, they more commonly identify alleles that may be markers for nearby pathologic variations-like a higher resolution version of the HLA haplotype. Compared to whole genome or exome sequencing, GWAS are a relatively inexpensive and quick way to identify variation across the genome that may be associated with disease.

Despite extensive use of this technology in other fields, no full genome array data have been reported for PD. One study looked at 9 susceptibility loci for DD in men with PD, of which only one was found to be associated with PD (rs4730775 in the WNT2 gene) (22). This finding was interesting, however, particularly given that WNT-signaling proteins are higher in PD plaques than adjacent normal tunica (23).

Consistent with a role of aberrant WNT signaling, TGF- $\beta 1$ (an activator of the WNT signaling pathway) is used to induce myofibroblast proliferation and plaque formation in animal models of PD $(17,24)$. Inhibition of TGF- $\beta 1$ signaling by overexpression of SMAD7 or silencing of HDAC2 or HDAC7 prevents myofibroblast transformation and progression of PD (24-26). Hauck et al. therefore hypothesized that SNPs in the TGF- $\beta 1$ gene might be associated with PD (27), but no significant associations were found.

\section{Karyotypic abnormalities}

Cultures of plaque-derived fibroblasts have been used by many laboratories to better understand the mechanisms leading to PD. Somers et al. discovered that plaque-derived fibroblasts from over half the men in their study had an abnormal karyotype, whereas none of the cultures derived from their adjacent normal tunica albuginea, skin, or lymphocytes were abnormal (28). Likewise, no abnormal karyotypes were found from non-PD scar- and chordeederived fibroblasts (28). A subsequent study confirmed a high rate of abnormal karyotypes in plaque-derived fibroblast cultures (9 of 14 men with PD) (29). Mulhall et al. also demonstrated high aneuploidy rates in $\mathrm{PD}$ plaquederived fibroblasts that were not observed in foreskin- or congenital curvature-derived fibroblast cultures (30).

Closer examination of the above data, however, indicates that genetic instability rather than specific karyotype abnormalities may lead to development of PD. First, in most of the studies, fibroblasts from normal tunica and lymphocytes came from the men with PD. Thus, it was only the plaquederived fibroblasts and not all the cells in their bodies that had 
the abnormal karyotype. Second, there is no consistent pattern to the karyotype abnormalities. Somers et al. found loss of the $Y$ chromosome in 3 of 9 cultures, while Guerneri found either a gain or loss of $\mathrm{Y}$ in 8 of 9 cultures $(28,29)$. Mulhall found an increased loss of $\mathrm{Y}$ as well as abnormalities in all other markers tested (i.e., 7, 8, 17, 18 and $\mathrm{X}$ ), while Guerneri found no trisomy 7 and 8 (29,30). Along these lines, Perinchery et al. demonstrated that PD plaques exhibit a high level of microsatellite instability and loss of heterozygosity compared to adjacent non-plaque tunica albuginea, lymphocytes and tunica albuginea from controls (31).

Additionally, it is notable that Mulhall and colleagues reported increasing karyotype abnormalities with higher passage numbers for plaque-derived and adjacent normal tunica-derived fibroblasts, but that the plaque-derived fibroblasts show instability at lower passage numbers (30). They subsequently demonstrated that the PD plaquederived fibroblasts developed into tumors when injected into nude mice (32). While these data may suggest that malignant transformation of fibroblasts could be causative in the pathogenesis of $\mathrm{PD}$, the rare occurrence of tunical sarcomas in $\mathrm{PD}$ would suggest that malignant transformation occurs more in vitro than in vivo.

One major issue, however, is that PD plaque-derived "fibroblast" cultures contain a high proportion of myofibroblasts, whereas normal tunica- and foreskinderived fibroblast cultures do not (33). Thus, whether the microsatellite instability or karyotypic abnormalities lead to the disease directly, or whether these are simply due to inherently different properties of myofibroblasts compared to fibroblasts in culture has yet to be determined.

Interestingly, myofibroblast infiltration is also found in DD nodules. Cytogenetic studies from DD nodule-derived cultures also show a high rate of chromosomal abnormalities, with trisomy 7, trisomy 8 , and loss of $\mathrm{Y}$ being reported in some studies $(34,35)$. Similar to PD plaque-derived fibroblast cultures, however, not all studies found the same chromosome abnormalities. Casalone et al. also reported high rates of aneuploidy, but trisomy 7 and 8 were only sporadically observed (36). Thus, while both PD and DD appear to share similar etiologies, the specific contribution of genetic instability remains unclear in both diseases.

\section{Gene expression changes in PD}

Several studies have focused on gene expression abnormalities in PD plaques using both hypothesis-driven testing and microarrays [summarized in (37)]. As with the karyotype abnormalities, however, the different cell composition of the analyzed tissue makes interpretation difficult. For example, Thomas et al. found decreased expression of alternatively spliced IGF1 mRNA isoforms in PD plaques compared to adjacent unaffected TA (38). Microarray analysis of PD plaques demonstrated upregulation of pathways favoring plaque formation and downregulation of pathways that inhibited plaque formation (39). In both these studies, however, sequencing of the genes was not performed; thus, whether or not these altered expression levels are due to genetic differences remains unclear. Nonetheless, given that both the plaque and normal tunica both came from men with $\mathrm{PD}$, it is less likely that the difference is due to DNA sequence. Rather, it likely reflects cell type-specific differences in gene expression between myofibroblasts and fibroblasts.

\section{Hurdles and potential solutions}

As demonstrated in the literature review above, there is evidence for a genetic contribution to PD. While there has been rapid growth in our knowledge and advancement in technologies that can facilitate genetic discovery, the precise mechanisms have remained elusive for over 40 years. Two factors contributing to this challenge are reemphasized below:

(I) There may be more than one gene responsible. As shown in the HLA typing, the HLA-B group reactivity appears to be particularly prominent in familial cases of PD but not in sporadic cases. One potential explanation is that multiple genes could affect predisposition to PD. As in other genetic diseases, identifying mutations associated with disease is easier in families as affected individuals share the same mutation. A group of unrelated individuals is more heterogeneous. The increased noise from the contribution of different genes can greatly reduce the power to detect significant differences.

(II) There is incomplete penetrance. PD often occurs in the setting of trauma to the erect penis. The likelihood of developing PD increases as exposure to inciting events increase. Thus, mean age at presentation is typically $50-70$ years $(8,40)$, although some men may never have an inciting event. Thus, it may be difficult to select controls as a person without PD may still have a genetic predisposition. This is particularly troublesome if the prevalence of disease is high, as among Caucasian men. Misclassification of controls can greatly increase the number of male subjects 
Table 1 Potential characteristics to identify subtypes of PD

Demographics
Age
Ethnicity
Family history
Exposure prior to disease onset
Microtrauma during intercourse
Intracavernosal injection
Penile fracture
Exposure prior to disease onset
Microtrauma during intercourse
Intracavernosal injection
Penile fracture
Disease
Pain with onset of disease
Plaque size/location/calcification
Number of plaques
Degree of curvature
erectile dysfunction, autoimmune disease)
Roncomitersion

needed to achieve statistical significance.

Given the number of families with extended pedigrees of highly penetrant PD may be limited, how might we overcome these hurdles? The key goal is to reduce the noise in the data. While using genetically related individuals greatly facilitates this, there are other methods that can be considered.

First, starting with the assumption that more than one gene may increase the risk of developing $\mathrm{PD}$, subclassifying PD may help create more homogenous groups. For example, in the HLA study, Nyberg found that 7 of 9 of the related men with PD also had DD (i.e., 78\%) which is considerably higher than the $11 \%$ among the general PD population reported by Leffell $(6,11)$. Thus, one potential categorization would be to study men with both PD and DD. Another subcategory could be men who painlessly develop PD plaques. Other potential characteristics that could be used to subdivide the general PD population are listed in Table 1.

Along these lines, identifying alleles that confer a higher risk is easier than identifying those that confer a smaller risk. Unusual presentations or outliers may be particularly beneficial in this regard. For example, studying men who develop PD at a young age [e.g., before age 40 (8)], men who develop PD without a history of sexual activity, or men from ethnic backgrounds where PD is rare, may help identify high impact, low frequency genetic contributors. Once identified, the contribution of these alleles could be studied in the general PD population.

A second important consideration is the identification of appropriate controls. Many studies selected controls who self-reported that they did not have PD or assumed that men from the general population were free of disease. This is problematic as up to a third of men found to have PD are unaware that they have the disease (40). Thus, it is critical that physical examination be done on both the men with PD and the controls. Beyond this, however, it is also important to ensure that men who are selected for controls have had sufficient exposure that they would likely have already developed PD if they were susceptible. One way to do this would be to use only older men (e.g., 70- or 80-year-old men); however, there is no guarantee that they have had sufficient exposure to the inciting event. A better way, perhaps, would be to use a cohort of men who have had known exposures without developing PD (e.g., on intracavernosal injection therapy or who have undergone penile fracture repair).

An extension of this second point is to ensure that confounders are minimized between the groups being compared. As highlighted in the karyotypic abnormalities section, many of these studies compared plaque and normal tissue from the same individuals. This reduces the noise that the genetic background contributes to gene expression; however, it also greatly reduces the ability to identify genetic factors that contribute to PD plaque development. Thus, the resulting data more likely identify gene expression differences between myofibroblasts and fibroblasts. Comparing normal tunica from men with PD to normal tunica from appropriately selected controls would facilitate identification of genetic differences that predispose men to the disease. This also applies to ethnic backgrounds of subjects and controls, exposures of subjects and controls, and other potential confounders.

\section{Conclusions}

Multiple lines of evidence have suggested that there is a 
genetic contribution that predisposes men to developing PD. Nonetheless, the data remain highly controversial and the question persists: what is the link? Technological advances have greatly increased the resolution with which we can search the genome; however, simply increasing the data collected is likely insufficient to identify the genes responsible for PD. As with genetic discoveries for other diseases, enriching for the presence of disease alleles while minimizing confounders will be paramount to answer this critical question.

\section{Acknowledgments}

Funding: JSG's work was supported in part by NIH K12 DK083014 Multidisciplinary K12 Urologic Research (KURe) Career Development Program (awarded to Dolores J. Lamb) and the Winfield Scott Charitable Trust.

\section{Footnote}

Provenance and Peer Review: This article was commissioned by the Guest Editors (Larry I. Lipshultz, Alexander W. Pastuszak) for the focused issue "Contemporary Issues and Controversies in Men's Health" published in Translational Andrology and Urology. The article was sent for external peer review organized by the Guest Editors and the editorial office.

Conflicts of Interest: The focused issue "Contemporary Issues and Controversies in Men's Health" was commissioned by the editorial office without any funding or sponsorship. The author has no conflicts of interest to declare.

Ethical Statement: The author is accountable for all aspects of the work in ensuring that questions related to the accuracy or integrity of any part of the work are appropriately investigated and resolved.

Open Access Statement: This is an Open Access article distributed in accordance with the Creative Commons Attribution-NonCommercial-NoDerivs 4.0 International License (CC BY-NC-ND 4.0), which permits the noncommercial replication and distribution of the article with the strict proviso that no changes or edits are made and the original work is properly cited (including links to both the formal publication through the relevant DOI and the license). See: https://creativecommons.org/licenses/by-ncnd/4.0/.

\section{References}

1. Dibenedetti DB, Nguyen D, Zografos L, et al. A Population-Based Study of Peyronie's Disease: Prevalence and Treatment Patterns in the United States. Adv Urol 2011;2011:282503.

2. Miner MM, Seftel AD. Peyronie's disease: epidemiology, diagnosis, and management. Curr Med Res Opin 2014;30:113-20.

3. Paulis G, Romano G, Paulis A. Prevalence, psychological impact, and risk factors of erectile dysfunction in patients with Peyronie's disease: a retrospective analysis of 309 cases. Res Rep Urol 2016;8:95-103.

4. Rhoden EL, Riedner CE, Fuchs SC, et al. A crosssectional study for the analysis of clinical, sexual and laboratory conditions associated to Peyronie's disease. J Sex Med 2010;7:1529-37.

5. Nugteren HM, Nijman JM, de Jong IJ, et al. The association between Peyronie's and Dupuytren's disease. Int J Impot Res 2011;23:142-5.

6. Nyberg LM Jr, Bias WB, Hochberg MC, et al. Identification of an inherited form of Peyronie's disease with autosomal dominant inheritance and association with Dupuytren's contracture and histocompatibility B7 crossreacting antigens. J Urol 1982;128:48-51.

7. Ziegelbaum M, Thomas A Jr, Zachary AA. The association of Peyronie's disease with HLA B7 cross-reactive antigens. A case report of identical twins. Cleve Clin J Med 1987;54:427-30.

8. Levine LA, Estrada CR, Storm DW, et al. Peyronie disease in younger men: characteristics and treatment results. J Androl 2003;24:27-32.

9. Zargooshi J. Trauma as the cause of Peyronie's disease: penile fracture as a model of trauma. J Urol 2004;172:186-8.

10. Willscher MK, Cwazka WF, Novicki DE. The association of histocompatibility antigens of the $\mathrm{B} 7$ cross-reacting group with Peyronie's disease. J Urol 1979;122:34-5.

11. Leffell MS, Devine CJ Jr, Horton CE, et al. Nonassociation of Peyronie's disease with HLA B7 crossreactive antigens. J Urol 1982;127:1223-4.

12. Rompel R, Weidner W, Mueller-Eckhardt G. HLA association of idiopathic Peyronie's disease: an indication of autoimmune phenomena in etiopathogenesis? Tissue Antigens 1991;38:104-6.

13. Nachtsheim DA, Rearden A. Peyronie's disease is associated with an HLA class II antigen, HLA-DQ5, implying an autoimmune etiology. J Urol 1996;156:1330-4.

14. Hauck EW, Hauptmann A, Weidner W, et al. Prospective 
analysis of HLA classes I and II antigen frequency in patients with Peyronie's disease. J Urol 2003;170:1443-6.

15. Noss MB, Day NS, Christ GJ, et al. The genetics and immunology of Peyronie's disease. Int J Impot Res 2000;12 Suppl 4:S127-32.

16. Ferretti L, Fandel TM, Qiu X, et al. Tunica albuginea allograft: a new model of LaPeyronie's disease with penile curvature and subtunical ossification. Asian J Androl 2014;16:592-6.

17. Antoniassi T, Junior FNF, Spessoto LCF, et al. Antifibrotic effect of mycophenolate mofetil on Peyronie's disease experimentally induced with TGF-beta. Int J Impot Res 2019. [Epub ahead of print].

18. Ventimiglia E, Capogrosso P, Colicchia M, et al. Peyronie's disease and autoimmunity-a real-life clinical study and comprehensive review. J Sex Med 2015;12:1062-9.

19. Ralph DJ, Mirakian R, Pryor JP, et al. The immunological features of Peyronie's disease. J Urol 1996;155:159-62.

20. Stewart S, Malto M, Sandberg L, et al. Increased serum levels of anti-elastin antibodies in patients with Peyronie's disease. J Urol 1994;152:105-6.

21. Pastuszak AW, Rodriguez KM, Solomon ZJ, et al. Increased Risk of Incident Disease in Men with Peyronie's Disease: Analysis of U.S. Claims Data. J Sex Med 2018;15:894-901.

22. Dolmans GH, Werker PM, de Jong IJ, et al. WNT2 locus is involved in genetic susceptibility of Peyronie's disease. J Sex Med 2012;9:1430-4.

23. Ten Dam EPM, van Driel MF, de Jong IJ, et al. Glimpses into the molecular pathogenesis of Peyronie's disease. Aging Male 2019. [Epub ahead of print].

24. Kwon KD, Choi MJ, Park JM, et al. Silencing histone deacetylase 2 using small hairpin RNA induces regression of fibrotic plaque in a rat model of Peyronie's disease. BJU Int 2014;114:926-36.

25. Choi MJ, Song KM, Park JM, et al. Effect of SMAD7 gene overexpression on TGF-beta1-induced profibrotic responses in fibroblasts derived from Peyronie's plaque. Asian J Androl 2015;17:487-92.

26. Kang DH, Yin GN, Choi MJ, Silencing Histone Deacetylase 7 Alleviates Transforming Growth Factorbeta1-Induced Profibrotic Responses in Fibroblasts Derived from Peyronie's Plaque. World J Mens Health 2018;36:139-46.

27. Hauck EW, Hauptmann A, Schmelz HU, et al. Prospective analysis of single nucleotide polymorphisms of the transforming growth factor beta-1 gene in Peyronie's disease. J Urol 2003;169:369-72.
28. Somers KD, Winters BA, Dawson DM, et al. Chromosome abnormalities in Peyronie's disease. J Urol 1987;137:672-5.

29. Guerneri S, Stioui S, Mantovani F, et al. Multiple clonal chromosome abnormalities in Peyronie's disease. Cancer Genet Cytogenet 1991;52:181-5.

30. Mulhall JP, Nicholson B, Pierpaoli S, et al. Chromosomal instability is demonstrated by fibroblasts derived from the tunica of men with Peyronie's disease. Int J Impot Res 2004;16:288-93.

31. Perinchery G, El-Sakka AI, Angan A, et al. Microsatellite alterations and loss of heterozygosity in Peyronie's disease. J Urol 2000;164:842-6.

32. Mulhall JP, Martin DJ, Lubrano T, et al. Peyronie's disease fibroblasts demonstrate tumorigenicity in the severe combined immunodeficient (SCID) mouse model. Int J Impot Res 2004;16:99-104.

33. Mulhall JP, Anderson MS, Lubrano T, et al. Peyronie's disease cell culture models: phenotypic, genotypic and functional analyses. Int J Impot Res 2002;14:397-405.

34. Sergovich FR, Botz JS, McFarlane RM. Nonrandom cytogenetic abnormalities in Dupuytren's disease. N Engl J Med 1983;308:162-3.

35. Dal Cin P, De Smet L, Sciot R, et al. Trisomy 7 and trisomy 8 in dividing and non-dividing tumor cells in Dupuytren's disease. Cancer Genet Cytogenet 1999;108:137-40.

36. Casalone R, Mazzola D, Meroni E, et al. Cytogenetic and interphase cytogenetic analyses reveal chromosome instability but no clonal trisomy 8 in Dupuytren contracture. Cancer Genet Cytogenet 1997;99:73-6.

37. Herati AS, Pastuszak AW. The Genetic Basis of Peyronie Disease: A Review. Sex Med Rev 2016;4:85-94.

38. Thomas CG, Psarros C, Gekas A, et al. Alternative Splicing of IGF1 Gene as a Potential Factor in the Pathogenesis of Peyronie's Disease. In Vivo 2016;30:251-6.

39. Magee TR, Qian A, Rajfer J, et al. Gene expression profiles in the Peyronie's disease plaque. Urology 2002;59:451-7.

40. Mulhall JP, Creech SD, Boorjian SA, et al. Subjective and objective analysis of the prevalence of Peyronie's disease in a population of men presenting for prostate cancer screening. J Urol 2004;171:2350-3.

Cite this article as: Gabrielsen JS. Peyronie's disease: is it genetic or not? Transl Androl Urol 2020;9(Suppl 2):S262-S268. doi: 10.21037/tau.2019.10.21 